\title{
HEAVY METAL POLLUTION CONTROL METHOD BASED ON CHEMICAL SOLIDIFICATION TECHNOLOGY
}

\author{
JIA, Q. \\ North China University of Water Resources and Electric Power, Zhengzhou 450000, China
}

Henan Engineering Research Center of Water Pollution and Soil Damage Remediation Zhengzhou 450000, China

Henan Key Laboratory of Water Environment Simulation and Treatment Zhengzhou 450000, China

(e-mail: jiamoney@126.com; phone: +86-1760-371-6888)

(Received $18^{\text {th }}$ Mar 2019; accepted $17^{\text {th }}$ May 2019)

\begin{abstract}
In view of the harm caused by sludge in the ecological environment, this paper adopts the solidification treatment technology based on the principle of environmental ecological effect to analyze the heavy metal pollution sources in sludge. With the aid of the solidification technology and considering the physical and chemical blocking effects of heavy metals, it analyzes the effect of the solidification technology in treatment of heavy metals at different ratios. The results show that the smaller the particle size, the better the leaching of heavy metals in the sludge; and that the leaching rate is higher under the acidic condition than under the alkaline one. Through research it is found that the impact of heavy metals in the sludge on the environment is influenced by two factors - the controlled distance and the time, so a model can be constructed to predict the migration parameters of heavy metals in the sludge according to the influencing predictors. Then based on the migration parameters, this paper introduces the inverse problem of pollution source control and the control method to optimize the problem. Through the above discussion, it proposes an environmental-effect-oriented heavy metal pollution control method, that is, optimizing the solidified materials by converting the source control problem to the inverse problem of capacity control. In this way, the concentrations of heavy metals in the sludge after solidification treatment can be effectively controlled to meet the requirements of environmental effects.

Keywords: heavy metal pollution, ecological effect principle, heavy metal migration parameters, source control method, model prediction
\end{abstract}

\section{Introduction}

Sludge is an inevitable product of sewage treatment and has a great impact on the environmental and ecological balance (Crivello, 2002; Zhang et al., 2018). The heavy metals, microorganisms and organic impurities attached to the forming process make the sludge treatment extremely complicated. According to statistics (Shukla et al., 2004), the amount of sludge accounts for about $2 \%-7 \%$ of the total treatment volume of sewage, and the annual investment and operating costs of sludge treatment account for $25 \%-30 \%$ and $25-40 \%$ of the sewage treatment costs, it takes a large part of the investment, for this reason, many scholars at home and abroad have carried out a lot of research on it, and have proposed the treatment methods and research ideas for the comprehensive utilization of sludge from different degrees. The research of many scholars has carried out meticulous research on it from the perspectives of reduction, stabilization, harmlessness and resource utilization, however, research on the comprehensive impact of heavy metals in sludge on environmental and ecological effects is still rare (Hong et al., 2014; Lee et al., 2014; Maati et al., 2018; Wang et al., 2018). Based on the principle of environmental ecological effects, Alvarez et al. (2002) 
started from an environmental perspective, applied the solidification technology to convert it into a process of non-flowability or solid formation, regardless of whether chemical bonding occurs between the waste and the curing agent, through the separation effect of this technology, they achieved the objective to control the harm of heavy metal to environmental pollution (Olier and Cosgrove, 2004), and improved the quality of the ecological environment.

Solidification technology uses cement to solidify the waste and cement the particles in the waste through the product of the cement hydration reaction (Yan et al., 2011; Zheng and Zhang, 2018). After solidification, the sludge forms denser blocks that have lower water permeability, so that the heavy metals existing in the form of oxides, hydroxides, insoluble salts, etc. in the sludge are continuously extracted and filtered out by adjusting the $\mathrm{pH}$ and Eh values of the sludge during the water treatment process. In this way, the harm of sludge is reduced (Nishioka et al., 2000). Based on the above theories, this paper adopts solidification technology to ensure the solidification conditions of heavy metal by changing the chemical and physical conditions of the solidified body, and based on this, it controls the heavy metal pollution control methods by discussing the control mechanism of heavy metals. This paper mainly discusses the control methods of two heavy metal elements $\mathrm{Cu}$ and $\mathrm{Zn}$ under the ecological effect conditions. The experimental study in this paper can effectively remove heavy metals in sludge and optimize sludge solidification effects and methods.

\section{Materials and methods}

During water treatment process, $50 \%-80 \%$ of the heavy metals in the sewage is concentrated in the sludge (Whanger, 2002; Georgieva, 2017). The solidification treatment technology is used to improve the properties of the sludge, making it a useful soil engineering material. The solidification technology mainly treats heavy metals in sludge, reduces the risk of metal leaching and its environmental hazard by reducing the water permeability of solidified sludge (Ying and Fang, 2006). Solidification technology reduces the impact of heavy metals on the environment through physical and chemical effects, the following passages will verify the solidification technology for the treatment of heavy metals in sludge by analyzing the principles and methods based on physical and chemical mechanisms.

\section{Results}

\section{Physical blocking effect of solidification methods on heavy metals}

This experiment took samples from typical sections from the upstream and midstream reaches of the Tianjin Beitang Sewage River and the Dagu Sewage River. In order to ensure the continuity and representativeness of the samples, the sampling was conducted respectively in the wet season, the normal season, and the dry season; and a total of 36 sets of samples had been taken. Then, the Soil Physical and Chemical Analysis Method was adopted to classify and test the collected samples, and the classified samples were subject to solidification process. The solidified sludge is dried and then crushed to different particle sizes, and sieved for classification. Then, X-ray diffraction (Japan Spv019) is used to analyze whether it will cause the destruction of the integrity of the organic matters in the solidified sludge, that is, whether there is a 
possibility of failure using physical method to treat the samples. Experimental method adopts the heavy metal leaching method: cement, sludge and water are arranged in a ratio of 1:2:5 and 2:2:5, respectively. For the heavy metal leaching experiment, at a soilwater ratio of 1:10, the samples with different particle sizes are mixed with the leaching solution, stirred, and shaken at different frequencies, then stand for more than 30 minutes, vacuum filtered, and use spectrophotometer (Beijing Ruili WFX130) to determine the concentration of $\mathrm{Cu}$ and $\mathrm{Zn}$. The experimental results and data are shown in Table 1.

Table 1. Relationship between solidified sludge particles and heavy metal leaching

\begin{tabular}{c|c|c|c|c}
\hline Particle(mm) & \multicolumn{2}{|c|}{ Cu leaching rate(\%) } & \multicolumn{2}{c}{ Zn leaching rate(\%) } \\
\hline 0.06 & 18.8 & 16.8 & 27.8 & 25.8 \\
0.09 & 17.2 & 14.9 & 24.2 & 22.9 \\
0.52 & 13.8 & 12.7 & 18.8 & 16.7 \\
0.65 & 10.7 & 9.6 & 15.7 & 14.6 \\
0.82 & 8.5 & 7.3 & 10.5 & 9.3 \\
2.5 & 5.2 & 3.8 & 8.2 & 7.8 \\
3.8 & 3.1 & 2.2 & 6.8 & 6.2 \\
7.2 & 1.5 & 0.7 & 5.2 & 0.7 \\
\hline
\end{tabular}

\section{Chemical blocking effect of solidification methods on heavy metals}

The previous study shows that, the stabilizing effect of solidified sludge heavy metals is due to the $\mathrm{pH}$ of the solidified sludge itself, it produces an incompatible or complexing effect on heavy metals to enhance their stability. The chemical blocking experiment is mainly to clarify the buffering effect of the sludge on the environmental changes after solidification. It changes the influence of its own chemical properties on heavy metal leaching by setting the external acid-base environment. By setting the leaching solution with different $\mathrm{PH}$ value and Eh value, the leaching solution of this paper is prepared by using hydrochloric acid and sodium hydroxide solutions, and the designed experiment is carried out according to the experimental ratio method of the physical effect, and the final experimental data is shown in Table 2 and Figures 1-2.

Table 2. Leaching rate of heavy metals under different $p H$ values

\begin{tabular}{c|c|c|c|c|c|c}
\hline PH & \multicolumn{3}{|c|}{ Cu leaching rate(\%) } & \multicolumn{3}{|c}{ Zn leaching rate(\%) } \\
\hline 1 & 73 & 45 & 25 & 82 & 45 & 38 \\
2 & 46 & 32 & 15 & 67 & 32.8 & 28.8 \\
3 & 32 & 19 & 11 & 45 & 28.7 & 23.9 \\
4 & 28 & 13 & 8.7 & 31 & 21.8 & 18.7 \\
5 & 16 & 7.6 & 7.7 & 22 & 17.5 & 17.8 \\
6 & 8 & 6.2 & 6.2 & 13 & 13.4 & 12.9 \\
7 & 6 & 5.6 & 5.5 & 8.7 & 11.7 & 11.8 \\
8 & 5.6 & 4.8 & 4.7 & 6.3 & 8.9 & 7.9 \\
9 & 5.2 & 3.2 & 2.8 & 4.7 & 5.3 & 4.2 \\
10 & 4.6 & 2.8 & 2.7 & 7.8 & 7.2 & 7.1 \\
11 & 3.8 & 2.4 & 2.2 & 10.2 & 9.8 & 10.3 \\
12 & 2.2 & 1.9 & 1.7 & 12.4 & 12.3 & 12.4 \\
13 & 1.8 & 1.4 & 1.5 & 14.5 & 13.7 & 13.9
\end{tabular}


Through the study of solidification and leaching treatment of heavy metals by physical effects and chemical effects, the experimental results show that different particle sizes have different effects on solidification and leaching of heavy metals. The trend in Figures 3-4 shows that, the leaching rate of particle size at different $\mathrm{pH}$ values first decreases and then increases, with the increase of particle size, the ratio of leached heavy metals shows a decreasing trend. The existing form of heavy metals largely determines the $\mathrm{pH}$ value, the trend in Figures 1-2 shows that the chemical route for solidifying heavy metals is to change the external chemical conditions, and the leaching rate is higher when it is acidic.

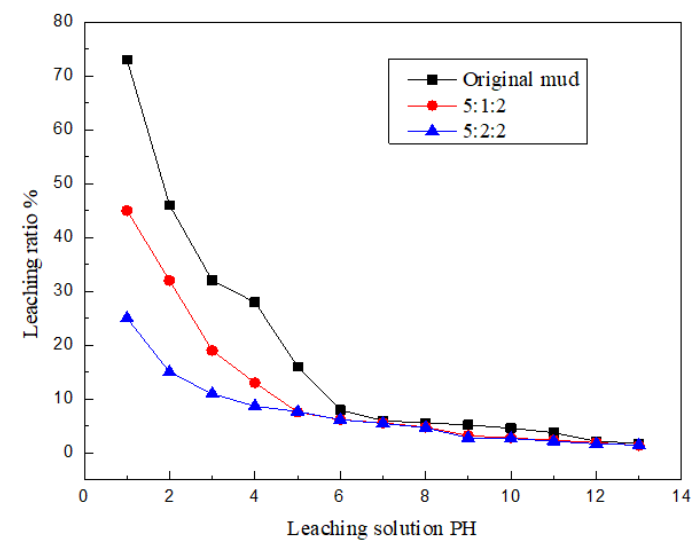

Figure 1. Leaching rate of $\mathrm{Cu}$ under different $\mathrm{PH}$ solidified sludge

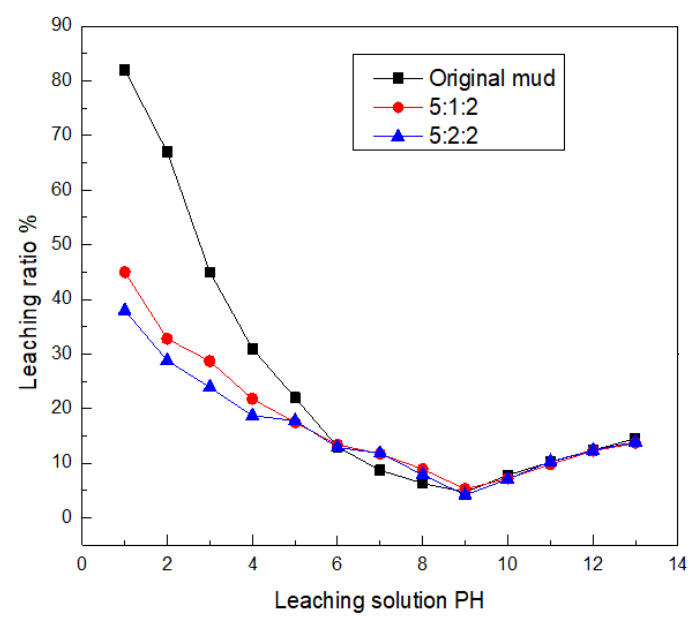

Figure 2. Leaching rate of Zn under different PH solidified sludge

\section{Discussion}

\section{Prediction of heavy metal pollution in sludge}

Assume after solidification, all the indicators of the sludge meet the standards of conventional soils (Xi-Shen et al., 2002), the model diagram of simulating the solidified sludge as the fill soil is as follows:

The mathematical formula of the above physical model is: 


$$
\operatorname{Rd} \frac{\partial \mathrm{C}}{\partial \mathrm{t}}=\mathrm{D}_{\mathrm{L}} \frac{\partial^{2} \mathrm{C}}{\partial \mathrm{x}^{2}}+\mathrm{DT} \frac{\partial^{2} \mathrm{C}}{\partial \mathrm{y}^{2}}-\mathrm{Vx} \frac{\partial \mathrm{C}}{\partial \mathrm{x}}-\mathrm{Vy} \frac{\partial \mathrm{C}}{\partial \mathrm{y}}
$$

where, $\mathrm{C}$ is the concentration of the substance, DL and DT are the vertical and horizontal dispersion coefficients, $\mathrm{Vx}$ and $\mathrm{Vy}$ are the flow rates in the $\mathrm{X}$ and $\mathrm{Y}$ directions, respectively. Using the above model and numerical method, the conditions of heavy metal sludge at different solidification levels are predicted (Philip et al., 2006), the prediction results are:

$$
\begin{aligned}
& D_{T}=a_{T} \frac{V^{2}{ }_{x}}{V}+a_{L} \frac{V^{2}}{V} \\
& D_{T}=a_{T} \frac{V^{2}{ }_{x}}{V}+a_{L} \frac{V_{y}^{2}}{V}
\end{aligned}
$$

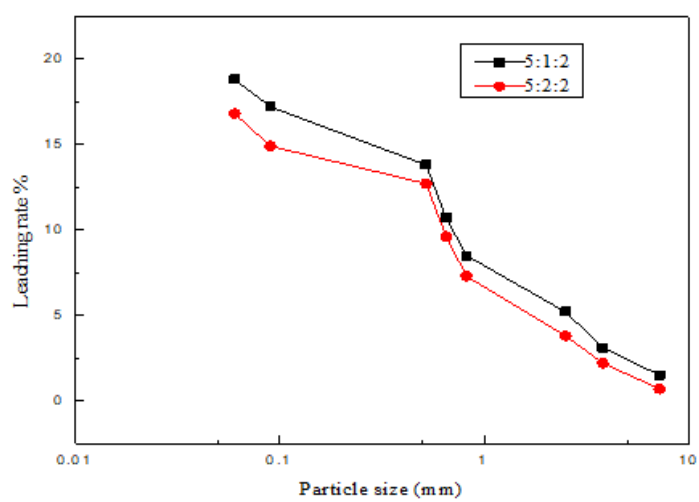

Figure 3. Leaching rate of $\mathrm{Cu}$ under different particle size solidified sludge

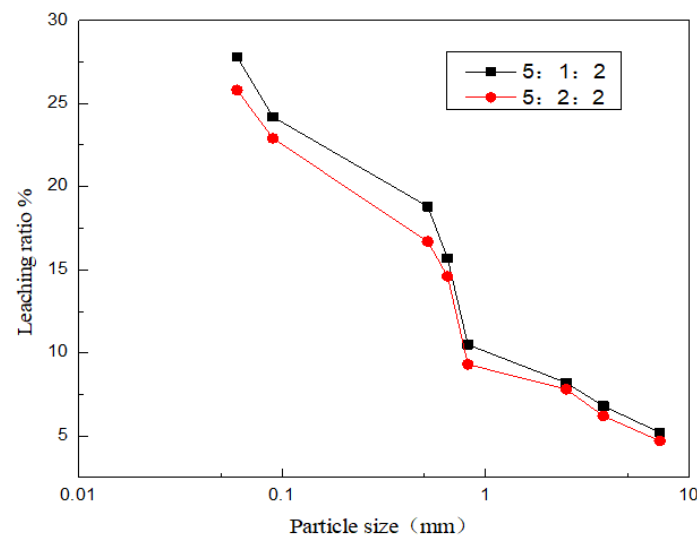

Figure 4. Leaching rate of Zn under different particle size solidified sludge

According to Eq. (1) - (3), calculate and analyze the parameters of the experimental materials listed in Table 3, and conduct experimental data simulation and calculation for 10 to 200 iterations, the final calculation results are shown in Table 4. 
Table 3. Experimental material parameters

\begin{tabular}{c|c|c|c|c|c}
\hline Material parameters & Slime & $\mathbf{5 : 1 : 1}$ & $\mathbf{5 : 1 : 2}$ & $\mathbf{5 : 2 : 3}$ & Surrounding medium \\
\hline Permeability coefficient & $1.25 \times 10^{-6}$ & $7.23 \times 10^{-7}$ & $8.27 \times 10^{-6}$ & $3.58 \times 10^{-6}$ & 5.25 \\
K $(\mathrm{cm} / \mathrm{s})$ & 0.031 & 0.065 & 0.081 & 0.105 & $\times 10^{-6}$ \\
Vertical dispersion al $(\mathrm{cm})$ & 8.92 & 11.5 & 12.6 & 14.1 & 87 \\
Block coefficient $\mathrm{R}_{\mathrm{d}}$ & 5.314 & 4.365 & 4.187 & 3.789 & 6.9 \\
Initial concentration C(mg/l) & 0.356 & 0.423 & 0.463 & 0.321 & 0 \\
Effective porosity $\mathrm{n}_{\mathrm{e}}$ & \multicolumn{2}{|c}{}
\end{tabular}

Table 4. Prediction of experimental data and results

\begin{tabular}{c|c|c|c|c}
\hline & Slime & $\mathbf{5 : 1 : 1}$ & $\mathbf{5 : 1 : 2}$ & $\mathbf{5 : 2 : 3}$ \\
\hline 10 & 4.25 & 4.16 & 2.89 & 2.72 \\
20 & 4.11 & 3.91 & 2.69 & 2.45 \\
30 & 3.89 & 3.79 & 2.22 & 1.89 \\
40 & 3.65 & 3.35 & 1.78 & 1.37 \\
50 & 3.25 & 3.05 & 1.58 & 1.09 \\
60 & 3.12 & 2.82 & 1.07 & 0.89 \\
70 & 3.08 & 2.48 & 0.75 & 0.68 \\
80 & 2.98 & 1.98 & 0.48 & 0.51 \\
90 & 2.76 & 1.74 & 0.32 & 0.31 \\
100 & 2.12 & 1.32 & 0.15 & 0.12 \\
110 & 1.78 & 1.18 & 0.06 & 0.03 \\
120 & 1.43 & 1.03 & 0.02 & - \\
130 & 1.22 & 0.92 & - & - \\
140 & 0.89 & 0.69 & - & - \\
150 & 0.74 & 0.34 & - & - \\
160 & 0.42 & 0.12 & - & - \\
170 & 0.31 & 0.031 & - & - \\
180 & 0.14 & 0.014 & - & - \\
190 & 0.08 & 0.008 & - & - \\
200 & 0.02 & 0.002 & & - \\
\hline
\end{tabular}

Using above theoretical model and numerical method, the diffusion coefficient of the sludge to the environment was calculated by Eq. (1), Eq. (2) and (3) respectively measure the pollution degree of different coordinate directions, and predict the heavy metal pollution of solidified sludge of different solidification levels, and the prediction was conducted based on principle of heavy metal migration parameters. This paper took the variation characteristics of $\mathrm{Zn}$ as an example to investigate the pollution law of heavy metals to the environment. Table 3 shows the parameters of the experimental materials.

According to the data in Tables 3 and 4 and the prediction analysis schemes, it can be known that: (1) The influence of heavy metals in the sludge on environmental pollution gradually increases with time, and the untreated sludge has a much larger groundwater pollution range than the sludge after solidification treatment, and the concentration is higher than the concentration of the sample after solidification; (2) The release rate of heavy metals in the solidified sludge is much smaller than that of untreated sludge, and the released heavy metals will also be diluted by groundwater to reduce the harm to the environment; (3) With the improvement of the solidification level, the pollution of heavy metals to the environment is gradually reduced, and the pollution of heavy metals can be controlled by increasing the solidification level. 


\section{Heavy metal migration parameters}

Based on the prediction method and control mechanism (Weng et al., 2001; Dragana et al., 2016), the permeability curve of heavy metals in solidified sludge was measured by a flexible permeation meter to obtain the diffusion degree and permeation structure coefficient of two heavy metal elements of $\mathrm{Cu}$ and $\mathrm{Zn}$. The test ratios are designed by changing the incorporation amount of cement and sludge, 5 ratios are set to perform the test, respectively are: $5: 1: 1,5: 1: 3,5: 2: 2,5: 3: 2$. According to the prediction results, the heavy metal concentration change values collected in different time periods are classified, and the maximum concentration value is taken as the initial concentration of heavy metals in the solidified sludge, and the calculated actual value of concentration is shown in Table 5.

The data in Table 5 and the trends of Figures 5-6 show that, the parameter results obtained based on the prediction are close the trend of heavy metals in the solidified sludge of the actual calculation result, so this test method can characterize the demand of the special pollutant parameters.

Table 5. Concentration of heavy metals in different days under different cement / sludge ratios

\begin{tabular}{c|c|c|c|c|c|c|c|c}
\hline \multirow{2}{*}{ days } & \multicolumn{4}{|c|}{ Zn migration concentration } & \multicolumn{4}{c}{ Cu migration concentration } \\
\cline { 2 - 9 } & $\mathbf{5 : 1 : 1}$ & $\mathbf{5 : 1 : 3}$ & $\mathbf{5 : 2 : 2}$ & $\mathbf{5 : 2 : 3}$ & $\mathbf{5 : 1 : 1}$ & $\mathbf{5 : 1 : 3}$ & $\mathbf{5 : 2 : 2}$ & $\mathbf{5 : 2 : 3}$ \\
\hline 1 & 4.8 & 3.7 & 3.6 & 3.2 & 0.46 & 0.36 & 0.39 & 0.34 \\
2 & 3.1 & 3.3 & 3.2 & 3.1 & 0.33 & 0.32 & 0.33 & 0.31 \\
3 & 0.9 & 2.8 & 2.7 & 2.8 & 0.12 & 0.18 & 0.26 & 0.25 \\
4 & 0.2 & 1.5 & 2.2 & 2.7 & 0.08 & 0.14 & 0.18 & 0.24 \\
5 & 0.02 & 0.6 & 1.9 & 2.4 & 0.03 & 0.09 & 0.12 & 0.19 \\
6 & - & 0.3 & 0.8 & 2.1 & - & 0.05 & 0.08 & 0.16 \\
7 & - & 0.15 & 0.25 & 1.7 & - & 0.03 & 0.06 & 0.11 \\
8 & - & 0.05 & 0.17 & 1.6 & - & 0.02 & 0.06 & 0.09 \\
9 & - & - & - & 1.3 & - & 0.01 & 0.02 & 0.06 \\
10 & - & - & - & 0.9 & - & - & - & 0.06 \\
15 & - & - & - & 0.3 & - & - & - & 0.03 \\
20 & - & - & - & 0.05 & - & - & - & 0.01 \\
\hline
\end{tabular}

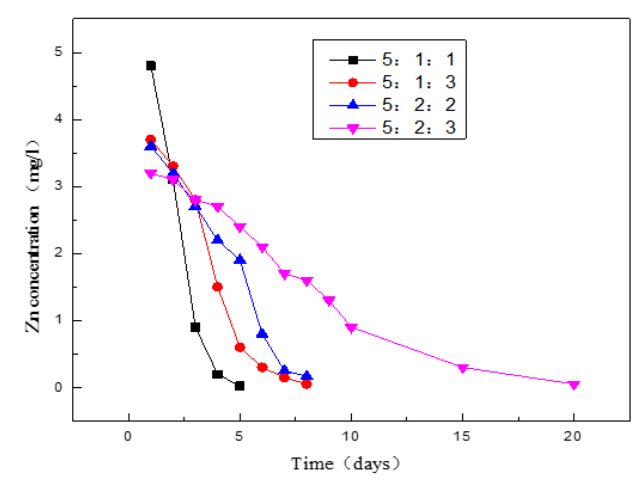

Figure 5. Concentration migration of heavy metal Zn under different proportions and days

According to Yao. et al. (2015) and the trend of Figure 6 we can know that, the pollution intensity of solidified sludge to the environment is mainly determined by the 
migration rate of the metal. The migration rate of the metal is determined by the ratio of cement and sludge, the dispersion degree of the sludge, the retardation coefficient of the metal, and the flow rate, etc. The concentration of heavy metals changes significantly with the migration time. The migration trend of different ratios shows that the concentration migration of heavy metals is most closely related to the number of days in the initial experiment. The migration ability of heavy metals is concentrated in the first 1-2 days. After 5 days, it tends to be balanced or cannot be detected anymore. Different ratios indicate that the migration rate is greater than the diffusion degree. Therefore, when the heavy metal control method is involved, the retardation coefficient of the migration medium should be emphasized.

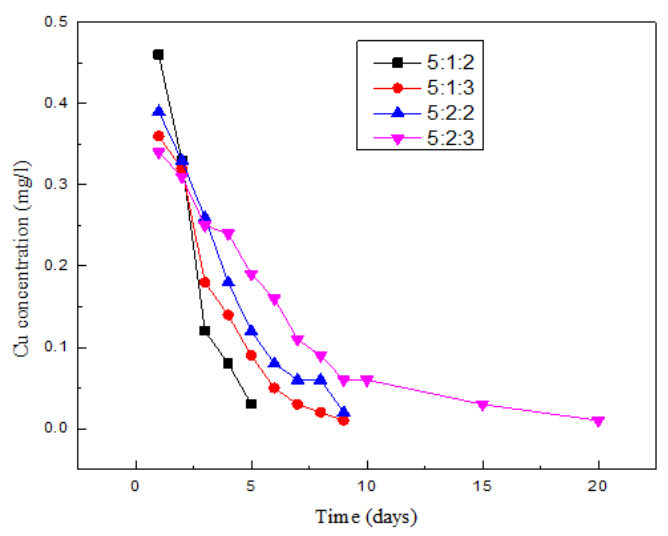

Figure 6. Concentration migration of heavy metal Zn under different proportions and days

\section{Heavy metal pollution control method}

It can be seen from the discussion that the pollutants contained in the sludge will inevitably enter the surrounding environment by penetrating into groundwater or surface runoff during the formation process, and the heavy metals is a potential danger source to the pollution of groundwater. The existing forms of heavy metals are mostly in a soluble state, and there are many hidden dangers of heavy metal pollution to the environment. According to the principle of environmental ecological effect, the theory of pollution source control reverse problem is used to investigate the concentration of metal in solidified sludge. (Yi et al., 2010) further discussed and optimized the solidified materials, and converted the problem of metal pollution control to the inverse and optimization problem of pollution source control.

To solve the inverse problem of the source item, we must first establish its mathematical model of the reverse source control (Plaia and Bondi, 2006), that is, select a calculation area, and use the solidified sludge as the filling material in the area, establish the model according to the prediction results of section 3.1, try to control the release of heavy metal concentration in the solidified sludge within the environmental tolerance range to meet the environmental capacity requirements.

In order to verify the above model, the solidified sludge sample is subjected to vacuum saturation treatment, and the concentration of heavy metals in the solidified sludge was determined by centrifugal separation method. After solidifying, vacuuming, and twice centrifugal separating, the concentration of heavy metals in pore water is detected, the results of repeated tests are tested for significance using regression 
equations, and the significant test results are consistent with predicted trends, therefore, the control method discussed above can control the pollution of heavy metals to the environment.

\section{Conclusions}

(1) The pollution control problem of heavy metals in solidified sludge can be transformed into source item control inverse problem and solidified material optimization problem. By quantifying the requirements of environmental capacity, we can limit the concentration of heavy metals in the solidified sludge. Through optimizing the solidification conditions and material parameters, we could obtain the pollution control scheme.

(2) Based on the principle of ecological environment effect, the sludge solidification treatment technology can measure the occurrence regularity of heavy metals by physical and chemical environment, and it can improve the leaching rate of heavy metals in the sludge, thereby improving the utilization effect of the sludge after solidification, so that it can meet the requirements of geomaterials.

(3) The control of heavy metal pollution is essentially a problem of source control, through centrifugation, separation and solidification technologies, we can control the pollution of heavy metals to the environment. The research results and methods of this paper can quantitatively reduce metal pollution by solidification technology and separation method.

Acknowledgements. Henan Science and Technology Project "Study on Biological Toxicity of Water Quality in Henan Province" (No.152102310343). Research Start up Fund of North China university of Water Resources and Electric Power (No:201621).

\section{REFERENCES}

[1] Alvarez, E. A., Mochón, M. C., Jiménez Sánchez, J. C., Ternero Rodríguez, M. (2002): Heavy metal extractable forms in sludge from wastewater treatment plants. Chemosphere 47(7): 765-775.

[2] Crivello, J. V. (2002): Advanced curing technologies using photo-and electron beam induced cationic polymerization. - Radiation Physics \& Chemistry 63(1): 21-27.

[3] Georgieva, V. (2017): Generalized net model of mechanical wastewater pre-treatment. International Journal Bioautomation 21(1): 133-144.

[4] Hong, S., Khim, J. S., Ryu, J., Kang, S. G., Shim, W. J. (2014): Environmental and ecological effects and recoveries after five years of the hebei spirit oil spill, taean, korea. - Ocean \& Coastal Management 102(275): 522-532.

[5] Lee, C. H., Lee, B. Y., Chang, W. K., Hong, S., Song, S. J., Park, J. (2014): Environmental and ecological effects of lake shihwa reclamation project in south korea: a review. - Ocean \& Coastal Management 102: 545-558.

[6] Maati, A., Ouakdi, E. H., Tabourot, L., Balland, P., Demouche, M. (2018): Modelling of the thermomechanical behaviour of FCC metals under various conditions. - Annales de Chimie - Science des Matériaux 42(1): 115-127.

[7] Nishioka, M., Yanagisawa, K., Yamasaki, N. (2000): Solidification of sludge ash by hydrothermal hot-pressing. - Research Journal of the Water Pollution Control Federation 62(7): 926-932. 
[8] Oliver, B. G., Cosgrove, E. G. (2004): The efficiency of heavy metal removal by a conventional activated sludge treatment plant. - Water Research 8(11): 869-874.

[9] Philip, L., Lyengar, L., Venkobachar, C. (2006): Immobilised microbial reactor for heavy metal pollution control. - International Journal of Environment \& Pollution 6(2-3): 277284.

[10] Plaia, A., Bondi, A. L. (2006): Single imputation method of missing values in environmental pollution data sets. - Atmospheric Environment 40(38): 7316-7330.

[11] Shukla, V., Bajpai, M., Singh, D. K., Singh, M., Shukla, R. (2004): Review of basic chemistry of uv-curing technology. - Pigment \& Resin Technology 33(5): 272-279.

[12] Wang, W., Zhang, K. F., Zhou, X. L., Wang, C. L., Huo, Z. K., Ye, P. F., Meng, X. Q. (2018): Deep reduction recovery of iron from copper slag. - Revue des Composites et des Matériaux Avancés 28(4): 539-549.

[13] Weng, L., Temminghoff, E. J., Riemsdijk, W. H. V. (2001): Contribution of individual sorbents to the control of heavy metal activity in sandy soil. - Environ.sci.technol. 35(22): 4436-4443.

[14] Whanger, P. D. (2002): Selenium in the treatment of heavy metal poisoning and chemical carcinogenesis. - Journal of Trace Elements \& Electrolytes in Health \& Disease 6(4): 209.

[15] Xi-Shen, Z., An-Huai, L. U., Xiang, G., Jin, Z., De-Sheng, Z. (2002): Contamination of heavy metals in soil present situation and method. - Soil \& Environmental Sciences 11(1): 79-84.

[16] Yan, J., Fanyong, S., Nanwen, Z., Tingting, G. (2011): Experiments on solidification of sewage sludge with different solidifying agents. - Environmental Pollution \& Control 33(2): 74-78.

[17] Yi, C., Li-Jiang, X., Xiao-Yong, Y. U. (2010): Identification method of rural environmental pollution and its application. - Journal of Agro-Environment Science 29(11): 2221-2227.

[18] Ying, X., Fang, Z. (2006): Experimental research on heavy metal wastewater treatment with dipropyl dithiophosphate. - Journal of Hazardous Materials 137(3): 1636-1642.

[19] Zhang, J. X., Sun, W. G., Niu, F. S., Wang, L., Zhao, Y. W., Han, M. M. (2018): Atmospheric sulfuric acid leaching thermodynamics from metallurgical zinc-bearing dust sludge. - International Journal of Heat and Technology 36(1): 229-236.

[20] Zheng, L., Xia, Z., Zhang, X. Y. (2018): Comparison between geopolymer reaction and cement hydration in solidification of fly ash generated in municipal solid waste incineration. - Revue des Composites et des Matériaux Avancés 28(3): 395-403. 